\title{
On the Fractional Chromatic Index of a Graph and its Complement
}

\author{
David Avis, ${ }^{1}$ Caterina De Simone ${ }^{2}$ and Bruce Reed ${ }^{1}$ \\ School of Computer Science, McGill University, \\ 3480 University Street, Montreal, Canada, H3A2A7. ${ }^{1}$ \\ Istituto di Analisi dei Sistemi ed Informatica (IASI), \\ CNR, Viale Manzoni 30, 00185 Rome, Italy. ${ }^{2}$
}

24 September 2004

\begin{abstract}
The chromatic index $\chi_{e}(G)$ of an undirected graph $G$ is the minimum number of matchings needed to partition its edge set. Let $\Delta(G)$ denote the maximum vertex degree of $G$, and let $\bar{G}$ denote the complement of $G$. Jensen and Toft conjectured that for a graph $G$ with an even number of vertices, either $\chi_{e}(G)=\Delta(G)$ or $\chi_{e}(\bar{G})=\Delta(\bar{G})$. We prove a fractional version of this conjecture.
\end{abstract}

\section{The Introduction}

The chromatic index $\chi_{e}(G)$ of a graph $G=(V(G), E(G))$ is the minimum number of matchings needed to partition its edge set (for the definition of matching and other standard terms in graph theory, see Bondy and Murty[1] ). Since all the edges incident to a vertex must be in different matchings, we know that $\chi_{e}(G)$ is at least the maximum degree of $G$, which we denote by $\Delta(G)$. In fact Vizing[5] proved that $\chi_{e}(G)$ is at most $\Delta(G)+1$, whilst Holyer[3] proved it is NP-complete to determine if $\chi_{e}(G)$ is $\Delta(G)$. If $H$ is a subgraph of $G$ with $2 t+1$ vertices, then we need at least $\lceil|E(H)| / t\rceil$ matchings to cover $E(H)$. Thus if $|E(H)|>t \Delta(G)$, then $H$ and hence $G$ have chromatic index $\Delta(G)+1$. A subgraph $H$ of $G$ is called overfull if it has an odd number of vertices and

$$
|E(H)|>\Delta(G) \frac{|V(H)|-1}{2 .}
$$

If $G$ is a regular graph with an odd number of vertices then $G$ is overfull, and its complement, $\bar{G}$, being regular, is also overfull. Thus for such graphs $\chi_{e}(G)=\Delta(G)+1$ and $\chi_{e}(\bar{G})=\Delta(\bar{G})+1$. In [4], Jensen and Toft conjectured that this could not happen for graphs with an even number of vertices. They conjectured that if $G$ has an even number of vertices then either $\chi_{e}(G)=\Delta(G)$ or $\chi_{e}(\bar{G})=\Delta(\bar{G})$. As evidence in support of this conjecture, we show that it is true 
for fractional edge colourings.

A fractional edge colouring of $G$ is a non-negative weighting $w($.$) of the set \mathbf{M}(G)$ of matchings in $G$ so that for every edge $e \in E(G)$,

$$
\sum_{M: e \in M} w(M)=1
$$

The weight $\alpha$ of this colouring is defined by

$$
\alpha=\sum_{M \in \mathbf{M}(G)} w(M),
$$

and in this case we say that $G$ has a fractional $\alpha$-edge colouring.

The fractional chromatic index, $\chi_{e}^{f}(G)$, of $G$ is the minimum $\alpha$ for which $G$ has a fractional $\alpha$-edge colouring. By dividing the weight function by $\alpha$, we see that $G$ has a fractional $\alpha$-edge colouring if and only if the vector $(1 / \alpha, \ldots, 1 / \alpha) \in R^{|E(G)|}$ is a convex combination of incidence vectors of matchings of $G$. It follows from Edmonds' characterization of the matching polytope[2] that $\chi_{e}^{f}(G)$ can be computed in polynomial time, and that in fact

$$
\chi_{e}^{f}(G)=\max \left\{\Delta(G), \max _{H \subseteq G, H \text { overfull }} \frac{2|E(H)|}{|V(H)|-1}\right\} .
$$

We will use the corollary that if $\chi_{e}^{f}(G)>\Delta(G)$ then $G$ must contain an overfull subgraph. We may now state our result.

The Theorem. Let $G$ be a graph such that $\chi_{e}^{f}(G)>\Delta(G)$ and $\chi_{e}^{f}(\bar{G})>\Delta(\bar{G})$, then either $G$ or $\bar{G}$ is overfull.

Since overfull subgraphs have an odd number of vertices, this yields:

The Corollary If $G$ has an even number of vertices then either $\chi_{e}^{f}(G)=\Delta(G)$ or $\chi_{e}^{f}(\bar{G})=\Delta(\bar{G})$.

\section{The Proof.}

Arguing by contradiction, we suppose there is a graph $G$ such that neither $G$ nor its complement is overfull, yet $\chi_{e}^{f}(G)>\Delta(G)$ and $\chi_{e}^{f}(\bar{G})>\Delta(\bar{G})$. We may choose an overfull subgraph $H$ of $G$ and an overfull subgraph $F$ of $\bar{G}$. To simplify the exposition, in what follows we let $G$, $F$ and $H$ also stand for their vertex sets where no confusion arises. Since the sum of the degrees of all vertices of a graph is twice the number of its edges, letting $d_{H}(v)$ be the degree of a vertex $v$ in $H$, we see that (1) is equivalent to

$$
\sum_{v \in H}\left(\Delta(G)-d_{H}(v)\right) \leq \Delta(G)-1 .
$$

and implies that

$$
|H| \geq \Delta(G)+1 \text { and }|F| \geq \Delta(\bar{G})+1 .
$$

We call $d e f_{G}(v)=\Delta(G)-d_{G}(v)$ the deficiency, of a vertex $v$ in $G$, and let $E_{G}(A, B)$ be the set of edges in $G$ with one endpoint in $A \subseteq V$ and one endpoint in $B \subseteq V$. With these definitions and using (2) and (3) we have

$$
\sum_{v \in H} \operatorname{def}_{G}(v)+\left|E_{G}(H, G-H)\right| \leq \Delta(G)-1 \leq|H|-2 .
$$

Similarly, in the complement $\bar{G}$, we have 


$$
\sum_{v \in F} \operatorname{def}_{\bar{G}}(v)+\left|E_{\bar{G}}(F, \bar{G}-F)\right| \leq \Delta(\bar{G})-1 \leq|F|-2 .
$$

The subgraphs $H$ and $F$ naturally partition the vertices of $G$ into four parts with cardinalities $a, b, c, d$, as defined by

$$
a=|H \cap F|, \quad b=|H-F|, \quad c=|F-H|, \text { and } d=|G-F-H| .
$$

This partition is illustrated in Table 1 . Let $n=|V(G)|$.

\begin{tabular}{c|c|c|} 
& \multicolumn{1}{c|}{$\mathrm{F}$} & $\mathrm{G}-\mathrm{F}$ \\
\cline { 2 - 3 } $\mathrm{H}$ & $\mathrm{a}$ & $\mathrm{b}$ \\
\cline { 2 - 3 } $\mathrm{G}-\mathrm{H}$ & $\mathrm{c}$ & $\mathrm{d}$ \\
\cline { 2 - 3 } & &
\end{tabular}

\section{Table 1: Partition of $G$ into four subsets}

We note that if $v \in F-H$ and $w \in H-F$ then $v w$ is either an edge of $E_{G}(H, G-H)$ or of $E_{\bar{G}}(F, G-F)$. The same statement holds when $v \in F \cap H$ and $w \in G-F-H$. This implies the inequality

$$
a d+b c \leq\left|E_{G}(H, G-H)\right|+\left|E_{\bar{G}}(F, G-F)\right| .
$$

For every vertex $v$ of $G$ we have $d_{G}(v)+d_{\bar{G}}(v)=n-1$ and so $\Delta(G)+\Delta(\bar{G}) \geq n-1$. Hence we can define the nonnegative integer $k$ by

$$
k=\Delta(G)+\Delta(\bar{G})-n+1 .
$$

We also have that for all $v$,

$$
k=\operatorname{def}_{G}(v)+d e f_{\bar{G}}(v)
$$

and so

$$
\sum_{v \in H} d e f_{G}(v)+\sum_{v \in F} d e f_{\bar{G}}(v) \geq \sum_{v \in H \cap F}\left(d e f_{G}(v)+\operatorname{def} f_{\bar{G}}(v)\right)=a k .
$$

Combining the inequalities (4)-(9) we obtain the key inequality:

$$
b c+a(d+k) \leq \Delta(G)+\Delta(\bar{G})-2=n+k-3 .
$$

Manipulating this inequality will give the desired result. By (3)

$$
2 a+b+c \geq \Delta(G)+\Delta(\bar{G})+2 .
$$

Combining with (10) we have

$$
b c+a(d+k) \leq 2 a+b+c-4
$$

Now if $b, c \geq 1$ then $b c \geq b+c-1$. If in addition $d+k \geq 2$ then

$$
b c+a(d+k) \geq b+c-1+2 a>2 a+b+c-4
$$

a contradiction.

The remaining cases to consider are when either $b=0$ or $c=0$ or $0 \leq d+k \leq 1$. We will need the following two observations. 
Observation 1: $|H|,|F| \leq|G|-2$.

Proof: We know by hypothesis that $|H| \neq|G|$. Suppose $|H|=|G|-1$. Let $w$ be the vertex of $G-H$. From (4) we have

$$
d_{G}(w)=|E(H, G-H)| \leq \Delta(G)-1-\sum_{v \in H} \operatorname{def}_{G}(v) .
$$

In fact $d_{G}(w) \leq \Delta(G)-2$. This follows immediately if $\operatorname{def}_{G}(v) \geq 1$ for some $v \in H$. Otherwise every vertex of $H$ has degree $\Delta(G)$. Since the total degree of $G$ must be even and $|H|$ is odd, $d_{G}(w)$ must have the same parity as $\Delta(G)$ and again $d_{G}(w) \leq \Delta(G)-2$. On the other hand, $d_{\bar{G}}(w) \leq \Delta(\bar{G})$, which combined with (7) and (8) gives

$$
k \geq \Delta(G)+d_{\bar{G}}(w)-n+1=\Delta(G)-d_{G}(w) \geq 2 .
$$

This also implies that $d_{G}(w) \geq \Delta(G)-k$. Since $F \cap H=F-w$,

$$
\begin{gathered}
\sum_{v \in F \cap H}\left(\operatorname{def}_{\bar{G}}(v)+\operatorname{def}_{G}(v)\right)+|E(H, G-H)| \geq k(|F|-1)+\Delta(G)-k \\
=k|F|+\Delta(G)-2 k \geq \Delta(\bar{G})+1+\Delta(G)-2 k+(k-1)|F|,
\end{gathered}
$$

where we used (3) to get the last inequality. Since $F$ is overfull, $|F| \geq 3$ and we have

$$
\sum_{v \in F \cap H}\left(d e f_{\bar{G}}(v)+\operatorname{def}_{G}(v)\right)+|E(H, G-H)| \geq \Delta(G)+\Delta(\bar{G})+k-2 \geq \Delta(G)+\Delta(\bar{G}) .
$$

By combining (4) and (5), we see that this cannot happen, giving the desired contradiction. By replacing $G$ by its complement, we obtain $F \leq|G|-2$.

Observation 2: $\min (\Delta(G), \Delta(\bar{G})) \geq n / 2-1$.

Proof: We assume that $\Delta(\bar{G}) \leq n / 2-3 / 2$ and derive a contradiction. Since $\Delta(G)+\Delta(\bar{G}) \geq n-1$ it follows that $\Delta(G) \geq n / 2+1 / 2$ and hence $|H| \geq n / 2+3 / 2$, while $|G-H| \leq n / 2-3 / 2$. Furthermore every vertex of $G$ must have degree at least $n / 2+1 / 2$ and so

$$
|E(H, G-H)| \geq|G-H|(n / 2+1 / 2-(|G-H|-1))=|G-H|(n / 2+3 / 2-|G-H|) .
$$

When $|G-H|=2$ this value is $n-1$ and when $|G-H|=n / 2-3 / 2$ it is $3 n / 2-9 / 2$. By Observation 1, we have $|H| \leq n-2$, i.e. $|G-H| \geq 2$, and so

$$
|E(H, G-H)| \geq \min (n-1,3 n / 2-9 / 2) \geq n-3 \geq|H|-1
$$

which violates (4).

We now return to our manipulation of (10) for the cases when $b=0$ or $c=0$ or $0 \leq d+k \leq 1$. First assume that $b=0$ and so $H \subseteq F$. By Observation 2 and (3) we have

$$
a=|F \cap H|=|H| \geq \Delta(G)+1 \geq n / 2,
$$

and so $a \geq b+c+d$. If $d+k \geq 3$ then $a(d+k) \geq 3 a \geq 2 a+b+c+d$ contradicting (11). If $d+k=2$ then $a(d+k)=2 a \geq a+b+c+d=n$ contradicting (10). Similarly we obtain contradictions if $c=0$ and $d+k \geq 2$.

It remains to settle the cases where $d+k=0$ or 1 . If $d=0$ then neither $b$ nor $c$ can be zero (or else either $G$ or $\bar{G}$ is overfull), and so $b c \geq b+c-1$. Hence if $d=0$ and $k=1$ then 


$$
b c+a(d+k) \geq a+b+c+d-1=n-1
$$

again contradicting (10).

If $k=0$ then by (8) $G$ is $\Delta(G)$-regular and $\bar{G}$ is $\Delta(\bar{G})$-regular. Since regular graphs with an odd number of vertices are overfull, this means that $n$ is even. We have by (7) that $\Delta(G)+\Delta(\bar{G})=n-1$, and so can assume that $\Delta(G) \geq n / 2$. Hence by (3) we have that $|H| \geq n / 2+1$ and so $|G-H| \leq n / 2-1$. On the other hand, Observation 1 gives $|G-H| \geq 2$, thus

$$
\left|E_{G}(H, G-H)\right| \geq|G-H|(n / 2-(|G-H|-1)) \geq n-2,
$$

where the last inequality is obtained by checking the two extremal values of $|G-H|$. Combining (4) and (12) gives the required contradiction and completes the proof of the theorem.

\section{References}

1. A. Bondy and U.S.R. Murty, Graph Theory with Applications, American Elsevier (1976).

2. J. Edmonds, "Maximum Matching and a Polyhedron with 0,1-Vertices," J. of Research of the National Bureau of Standards (B), 69, pp. 125-130 (1965).

3. I. Hoyler, "The NP-Completeness of Some Edge-Partition Problems," SIAM J. Computing, 10, pp. 713-717 (1981).

4. T.R. Jensen and B. Toft, Graph Coloring Problems, Wiley, New York (1995).

5. V.G. Vizing, "On an Estimate of the Chromatic Class of a p-graph (in Russian)," Diskret Analiz., 3, pp. 25-30 (1964). 